\title{
FEATURE
}

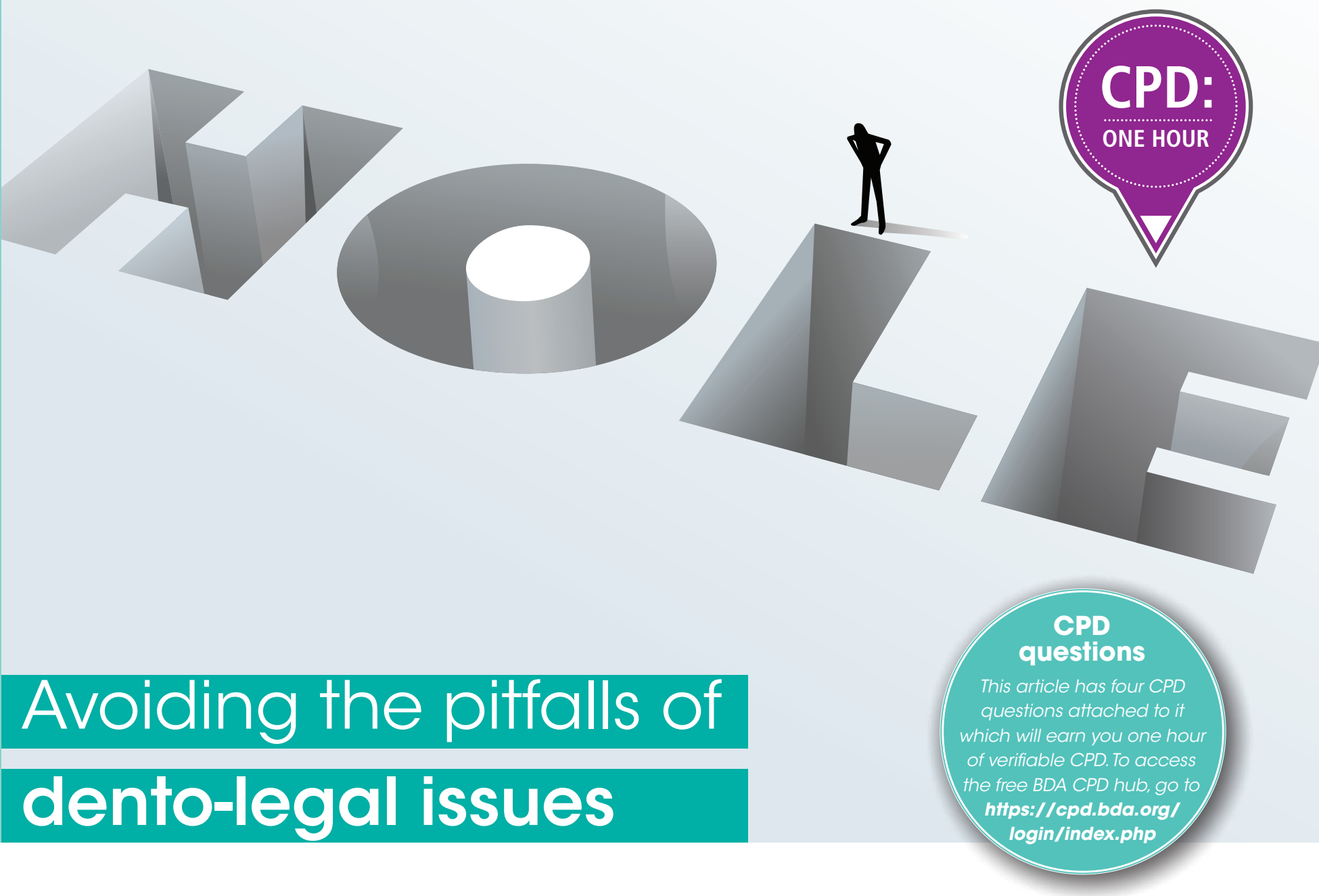

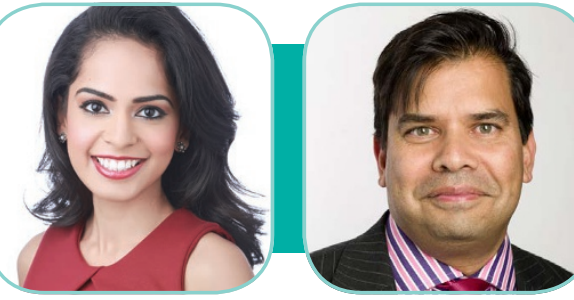

Introduction

Unfortunately your chances of being sued as a dental hygienist are higher than ever before. In the past five years, periodontal cases (the patients you see for the majority of your day) have soared up the leaderboard to claim the top spot. Whilst very few hygienists end up with claims or General Dental Council (GDC) investigations they get caught up with the dentists when the most common allegations of a failure to diagnose and failure to refer for specialist care are made. Implant cases are second on the leaderboard.

Periodontal cases are bad news for a number of reasons. Firstly, they tend to cover extended periods of time often stretching back many years involving several dentists and dental hygienists. Furthermore, when patients are able to demonstrate that they had not been advised of the presence of periodontal disease and have only learned this relatively recently (usually after seeing a different clinician), the

Dr Reena Wadia, Specialist Periodontist, and Dr Len D'Cruz, Medico-legal Advisor, provide a guide for dental hygienists.

normal three-year limitation period may be waived. When looking at the figures, they're worryingly high and continuing to increase. Most of these claims cost between $£ 25,000$ and $£ 75,000$ to settle but many of them cost $£ 100,000$ or more. ${ }^{1}$

\section{Record keeping}

Even when dental hygienists are adamant that they had told their patient over many years about their periodontal disease, discussed and given advice regarding oral hygiene, smoking cessation and other risk factors, and regularly monitored periodontal health, the clinical records often do not reflect these facts in sufficient detail (or at all), leaving them clearly disadvantaged.

Therefore, documentation is just as important as clinical management. Always discuss and record the diagnosis and consequences of untreated disease. When providing oral hygiene instructions, ensure you add in as much detail as possible; 'OHI given' is not enough. When describing levels of plaque control, use objective measurements such as plaque scores rather than subjective measures. Smoking cessation advice, where necessary, needs to have been provided and followed-up.

If abbreviations are used, they must be widely recognised. It can be helpful to create templates to save time and ensure key points are not missed out but these must be personalised to the patient. Here are a few helpful phrases you might want to include in your templates: ${ }^{2}$

- Patient advised of mild/moderate/severe periodontal disease

- Patient warned of tooth mobility and tooth loss related to periodontal disease

- Patient advised of smoking related to periodontal disease - increased risk factor for condition and poorer response to therapy 
- Patient advised that oral hygiene is not adequate to support formal periodontal therapy. Advised to improve plaque levels to support periodontal therapy

- Patient shown how to use interdental brushes properly and advised sizes...

- Discussed referral to periodontal specialist.

As well as verbal discussions, written information leaflets can be helpful. If these are given, ensure you make a record of this in your notes. If you face a difficult patient who is not complying, it sometimes helps to write them a letter explaining the importance of treatment and risks of not complying and reassuring them you are there to help when they are ready.

Part of enabling a clinician to follow a patient's response to the advice given is to record the patient attitudes, using their own words. This may be following advice about smoking cessation or initially checking if the patient is non-compliant. If this is clear from the notes the patient can hardly expect to win a claim for a failure to treat by the hygienist.

Increasingly as dental hygienists see direct access patients, the risks of claims are increased. Make sure you have the appropriate time to see these patients who may not have seen a dentist for a while. Make sure they are advised to see a dentist and record this advice in the clinical records, especially if you see any problems on the radiographs you take.

\section{Referring for specialist care}

It's important to be aware of the British Society of Periodontology (BSP) guidelines, ${ }^{3}$ which recommend referral for cases of complexity 3. This includes cases with a BPE score of 4 in any sextant as well as one or more of the following additional factors:

- Medical factor that is affecting the periodontal tissues, such as diabetes

- Complicating root morphologies and anatomical factors adversely affecting prognosis

- Aggressive periodontitis

- No response to previous optimally carried out treatment

- Patients requiring surgical procedures

- Medical history significantly affecting clinical management (history of head/ neck radiotherapy or intravenous bisphosphonate therapy)

- Immunocompromised/ immunosuppressed, significant bleeding disorder, potential drug interactions

- Regular tobacco smoking (10+ cigarettes/ day)

- Concurrent mucogingival disease such as erosive lichen planus.
If the patient refuses referral, make sure they understand the consequences of what they are not accepting. For example, if you don't have further treatment, the disease may progress, you may lose teeth and end up needing dentures.

\section{Implants}

As dental hygienists you will be seeing the same patients on a regular basis and many of them will have dental implants. If you are unable to detect problems around implants, in-house, limiting the damage caused by the complaint. Usually patients are just seeking an apology, explanation and reinstatement or intent to remedy the situation.

Audit the types of complaints you get and put systems in place to ensure that the same problem doesn't arise for other patients.

If you do have to go through a GDC claim, it can be very stressful and lead to sleepless nights so ensure you are supported by the right people and always follow the advice provided by your indemnity organisation.

\section{'INCREASINGLY AS DENTAL HYGIENISTS SEE}

\section{DIRECT ACCESS PATIENTS, THE RISKS OF CLAIMS}

ARE INCREASED. MAKE SURE YOU HAVE THE

\section{APPROPRIATE TIME TO SEE THESE PATIENTS WHO}

\section{MAY NOT HAVE SEEN A DENTIST FOR A WHILE.'}

you may be at high risk of a claim if the patient does end up later finding out the implant has failed.

Many hygienists are reluctant to examine dental implants, as they are not sure what they are looking for and worried about causing damage. It is essential that you gently probe all dental implants, as this is a key method by which you will pick up any signs of periimplant disease. You can use a regular metal probe and should record a four- or six-point pocket chart. A BPE is not appropriate for implants. Look out for bleeding, suppuration and an increasing probing depth. You may also require a radiograph to assess the bone levels.

If you detect any issues, refer the patient to the appropriate dentist/specialist. Early referral is key as cases of peri-implantitis can progress rapidly.

\section{Keep your energy levels in check} It has been found that most people make mistakes or miss things when they are tired or over-worked. Ensure you plan in regular breaks from work throughout the year to reenergise at the right time.

\section{How to manage a complaint}

Aim to deal with any complaints as quickly as possible. Be aware of the practice complaints procedure and get in touch with your indemnity organisation if required. Always try to resolve the patient's dissatisfaction

\section{Conclusion}

An increased awareness of how frequent these cases are becoming and a proactive approach through some of the simple steps described will significantly reduce your risk as a dental hygienist.

1. Willatt S. Dental implant feature: Costly claims. Riskwise 49: 14-16. Available at: https://www.dentalprotection.org/docs/ librariesprovider4/default-documentlibrary/riskwise-uk.pdf?sfvrsn=0 (accessed 19 April 2018).

2. Saleem S. Healthy gums do matter! First edition. Greater Manchester LDN, 2014.

3. British Society of Periodontology. Referral policy and parameters of care 2011.

Available at: https://www.bsperio.org. uk/publications/downloads/28_143801_ parameters_of_care.pdf (accessed 19 April 2018)

\section{CPD questions}

This article has four CPD questions attached to it which will earn you one hour of verifiable CPD. To access the free BDA CPD hub, go to https:// cpd.bda.org/login/index.php 